\title{
Cathepsin S-mediated autophagic flux in tumor-associated macrophages accelerate tumor development by promoting M2 polarization
}

Min Yang ${ }^{1,2+}$, Jingwei Liu ${ }^{1,2+}$, Jianghua Shao ${ }^{3}$, Yanwen Qin ${ }^{1,2}$, Qunsheng $\mathrm{Ji}^{4}$, Xiaolin Zhang ${ }^{4}$ and Jie Du ${ }^{1,2^{*}}$

\begin{abstract}
Background: Tumor-associated macrophages (TAMs) are the major component of tumor-infiltrating leukocytes. TAMs are heterogeneous, with distinct phenotypes influenced by the microenvironment surrounding tumor tissues, but relatively little is known about the key molecular in these cells that contribute to malignant phenotypes. Autophagic activity is a critical factor in tumor development that contributes to enhancing cellular fitness and survival in the hostile tumor microenvironment. However, the molecular basis and relations between autophagy and TAMs polarization remain unclear.
\end{abstract}

Methods: Cathepsin S (Cat S) expression was analyzed in human colon carcinoma and normal colon tissues. In vivo effects were evaluated using PancO2 subcutaneous tumor model and SL4 hepatic metastasis model. Immunofluorescence staining, flow cytometry and real-time PCR were done to examine TAMs polarization. Western blotting assay, transmission electron microscopy, mCherry-GFP-LC3 transfection and DQ-BSA degradation assays were carried out to determine its role in regulating autophagy.

Results: In the present study, we showed that the enhanced expression of Cat S correlated with the severity of histologic grade as well as clinical stage, metastasis, and recurrence, which are known indicators of a relatively poor prognosis of human colon carcinoma. Cat $\mathbf{S}$ knockout led to decreased tumor growth and metastasis. Moreover, Cat S knockout inhibited M2 macrophage polarization during tumor development. We further demonstrated that Cat $\mathrm{S}$ was required for not only autophagic flux but also the fusion processes of autophagosomes and lysosomes in TAMs. Importantly, we found that Cat S contributed to tumor development by regulating the M2 phenotype of TAMs through the activation of autophagy.

Conclusions: These results indicated that Cat S-mediated autophagic flux is an important mechanism for inducing M2-type polarization of TAMs, which leads to tumor development. These data provide strong evidence for a tumor-promoting role of autophagy in TAMs and suggest Cat $S$ could be a potential target for cancer therapy.

Keywords: Tumor-associated macrophages, Tumor microenvironment, Cathepsin, Autophagy

\footnotetext{
* Correspondence: jdu@bcm.edu

${ }^{\dagger}$ Equal contributors

'Beijing Anzhen Hospital, Capital Medical University, Beijing, China

${ }^{2}$ The Key Laboratory of Remodeling-Related Cardiovascular Diseases, Capital Medical University, Ministry of Education, Beijing Institute of Heart Lung and Blood Vessel Diseases, Beijing 100029, China

Full list of author information is available at the end of the article
} 


\section{Background}

Smouldering inflammation is a component of the tumor microenvironment, has recently been considered a hallmark of cancer with an important role in tumor initiation and progression [1,2]. In tumor microenvironment, innate immune cells are highly represented, and among the most abundant of these are macrophages. Although the original hypotheses proposed that macrophages are involved in antitumor immunity, there is substantial clinical and experimental evidence that in the majority of cases these tumor-associated macrophages (TAMs) enhance tumor progression to malignancy [3]. TAMs are heterogeneous in response to environmental signals and generally exhibit similarities with prototypic polarized M2 macrophages, contributing to tumor growth, invasion and angiogenesis [3-5]. Human clinical studies have shown a role of TAMs as tumor promoters based on the association of increased density of TAMs with tumor vascularization, metastasis, and poor prognosis [6-10], which have served as a paradigm for cancer-related inflammation. The link between TAMs and tumor development is well established. However, the mechanisms of TAMs moving toward a tumor-promoting phenotype are not fully understood.

Autophagy is an evolutionarily conserved, catabolic process that involves the entrapment of cytoplasmic components within characteristic vesicles for their delivery to and degradation within lysosomes [11-13]. The role of autophagy extends beyond the general homeostatic removal, degradation, and recycling of damaged proteins and organelles to many specific physiological and pathological processes such as development, immunity, energy homeostasis, cell death, tumorigenesis, among others [14-16]. Autophagy is a multifaceted process, and alterations in autophagic signaling pathways are frequently found in cancer [17-19]. While the involvement of autophagy in tumor development is widely accepted, it remains incompletely understood.

It is generally suggested that endolysosomal proteases play important roles in the degradation and regulation events of autophagic processes [11]. Cathepsins are cysteine lysosomal proteases that are essential for the turnover of intracellular and extracellular proteins internalized by endocytosis; cathepsins are now recognized that cysteine proteases play pivotal roles in cancer progression [20]. Of the cysteine cathepsins, B, L and S have been implicated most in serving as prognostic markers in cancer associated with poor outcome [21-25]. Cathepsin S (Cat S), unlike the ubiquitous cathepsin B (Cat B) and cathepsin L (Cat L), exhibits a restricted tissue expression. It is found predominantly in lymphatic tissue, macrophages, and other antigen-presenting cells [26]. There is an increasing body of data highlighting the upregulation of Cat $\mathrm{S}$ in a spectrum of tumors [23-25,27], where levels of the protease increase with the grade and aggressiveness of disease $[28,29]$. Studies previously demonstrated that macrophagesecreted Cat S plays a key role in tumor progression [30]. Recently, we further elucidated that Cat S deficiency results in abnormal accumulation of autophagosomes in macrophages and enhances Angiotensin II-induced cardiac inflammation [31]. However, whether Cat S-mediated autophagic flux promotes tumor development via the induction of TAMs polarization remains unclear. Thus, understanding the complex catabolic reactions that occur in the endolysosomal compartment is crucial for elucidating the mechanisms underlying TAMs-mediated tumor-promoting effects.

In this study, we identified that the expression of Cat $\mathrm{S}$ by TAMs is critical for promoting tumor growth and metastasis in vivo. We showed that Cat S deletion significantly blocked polarizing macrophages to the M2 phenotype within the tumor microenvironment. Moreover, we observed that Cat S deficiency led to the accumulation of autophagosomes and attenuation of autophagic flux in macrophages within the tumor microenvironment. Finally, we demonstrated that Cat S-mediated autophagic flux was pivotal to maintain polarization of the M2 TAMs phenotype.

\section{Results}

\section{Cat $\mathbf{S}$ expression is elevated in human cancer tissue}

To determine whether cathepsin levels are increased in human colon carcinoma, real-time quantitative PCR was performed to measure the expression of cathepsin family members in human colon carcinoma and adjacent normal colon tissues. As shown in Figure 1A, the mRNA levels of Cat B, Cat L and Cat S were significantly elevated in cancer specimens compared with the matched normal tissues. Especially, analysis by quantitative real-time PCR revealed Cat $\mathrm{S}$ was prominently expressed and highly enriched in colon carcinoma specimens compared to healthy tissues, while the mRNA expression of Cat D, Cat F, Cat H, Cat $\mathrm{K}$ or $\mathrm{Cat} \mathrm{Z}$ was not significant difference between colon carcinoma and normal colon tissues (Figure 1A). Immunohistochemical staining demonstrated that the expression of Cat $\mathrm{S}$ in adjacent normal colon tissues was low, whereas Cat $\mathrm{S}$ was observed in inflammatory-like cells located in carcinoma tissues (Figure 1B, left panels). Cat $\mathrm{S}$ expression was significantly elevated in poorly differentiated colon carcinoma, which was markedly higher than that of moderately differentiated or well differentiated colon carcinoma (Figure 1B, left panels). Moreover, we observed that Cat $S$ was expressed in a high percentage of stage III and IV tumors (Figure $1 \mathrm{~B}$, right panels). Analysis of Cat $\mathrm{S}$ expression in primary tumors and metastases formation revealed that patients who had advanced clinical stage disease, metastasis, or recurrence within 3 years showed increased Cat $\mathrm{S}$ expression in colon cancer tissue 


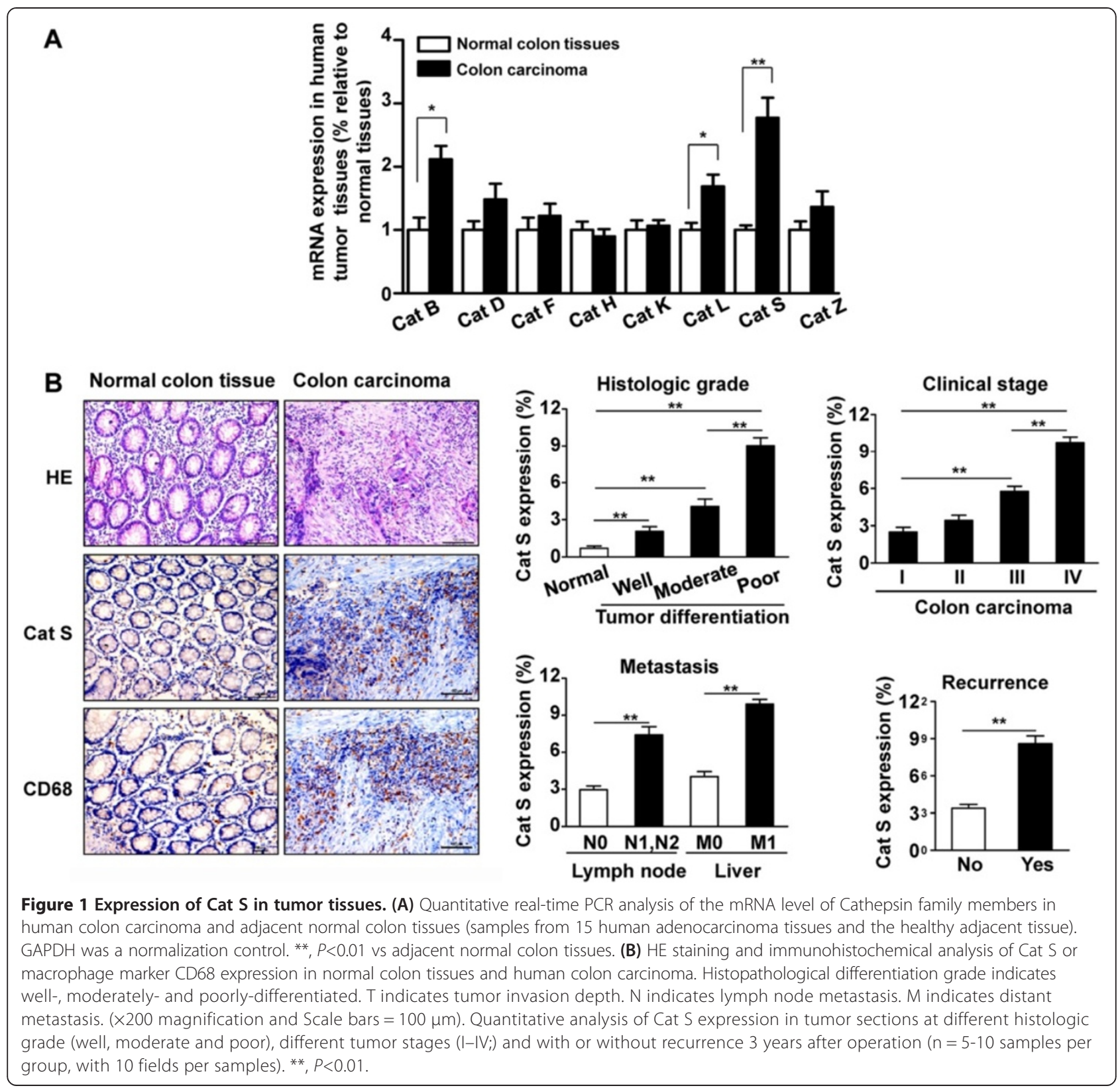

(Figure 1B, right panels). Thus, Cat S expression correlates with human colon cancer aggressiveness.

Furthermore, advanced colon carcinoma tissues were markedly and extensively infiltrated by CD68-positive macrophages, particularly along the tumor cell-invasive front, while few macrophages were observed in normal colon tissues (Figure 1B, left panels). Cat S was expressed mainly in stromal macrophages as verified by staining with CD68, demonstrating that Cat S localizes to macrophages in stromal compartments of solid tumors. Cat S-positive cells within the area colocalized with CD68-positive macrophages, indicating a high concordance of CD68 and
Cat $\mathrm{S}$ expression in human primary colon carcinoma during malignant progression.

Cat S deficiency inhibits tumor growth and metastasis In order to investigate the roles of Cat $S$ in the tumor microenvironment, we analyzed the effects of Cat S knockout on tumor growth and metastasis using subcutaneous and metastatic tumor models. In $\mathrm{PancO}_{2}$ subcutaneous tumor model, we found that Cat S knockout substantially suppressed tumor volume and reduced tumor weight (Figure 2A). The average tumor volume in the WT mice increased to $495.3 \pm 73.17 \mathrm{~mm}^{3}$ for 28 days after $\mathrm{PancO}_{2}$ 


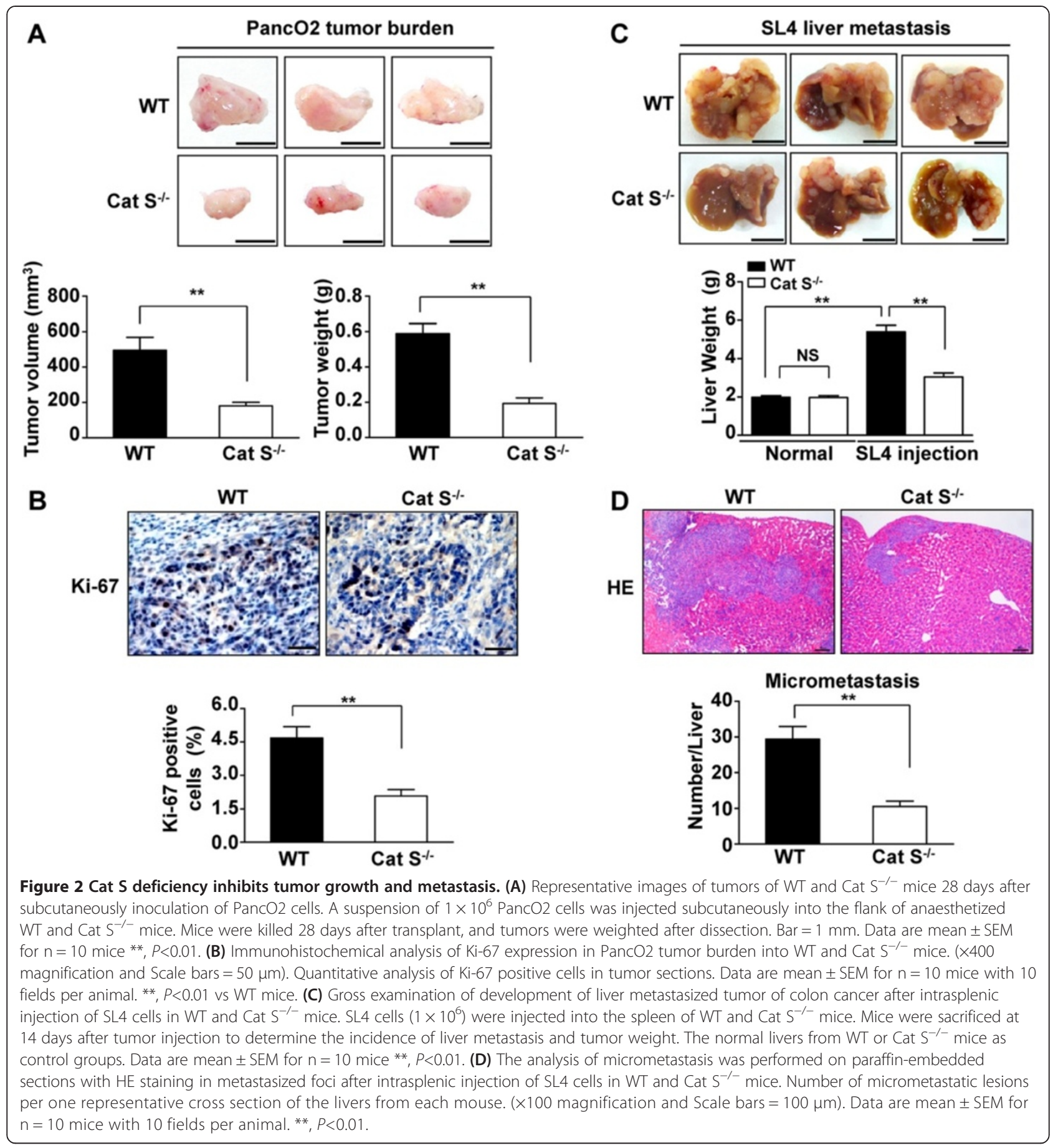

cells subcutaneous injection, whereas that in Cat $\mathrm{S}^{-/-}$mice decreased to $181.0 \pm 20.06 \mathrm{~mm}^{3}$, a significant inhibition of tumor growth $(P<0.01)$. Moreover, immunohistochemistry showed that $\mathrm{Ki}-67$ expression in PancO2 subcutaneous tumors was significantly lower in Cat $\mathrm{S}^{-1-}$ mice than in WT mice (Figure 2B). Previous studies have shown that SL4 cells injected intrasplenically formed tumors in the livers of mice [32], thus the SL4 cell line is a useful tool to study the process of tumor metastasis. To investigate the role of Cat S in liver metastasis of colon carcinoma cells, SL4 cells were injected into the spleens of WT and Cat $\mathrm{S}^{-/-}$mice.

As shown in Figure $2 \mathrm{C}$, there was no difference in normal liver weight between WT and Cat $\mathrm{S}^{-1-}$ mice. Fourteen days after SL4 injection, gross inspection demonstrated a marked increase of liver weight in WT mice due to multiple hepatic tumor nodules compared with weight of normal liver, whereas livers from Cat $\mathrm{S}^{-/-}$mice showed fewer 
tumor foci and decreased tumor-occupied weight relative to that in WT mice (Figure $2 \mathrm{C}$ ). To further investigate whether Cat S knockout attenuates tumor metastasis, the metastatic potential was quantified by scoring micrometastasis to livers in H\&E-stained sections. We observed the number of metastatic foci was markedly decreased in Cat $\mathrm{S}^{-/-}$mice versus WT mice on HE staining (Figure 2D), indicating a strong suppression in the metastatic potential of tumor cells. To investigate whether suppressing tumor development in Cat $\mathrm{S}^{-/-}$mice could be caused by inhibition of angiogenesis, the sections were immunostained using the CD31 antibody (a marker of endothelial cells) to assess angiogenesis in tumor tissues. As shown in Additional file 1: Figure S1A, immunostaining for endothelial cells (CD31) demonstrated reduced microvessel density in $\mathrm{PancO} 2$ subcutaneous tumor from Cat $\mathrm{S}^{-/-}$mice compared with that of in WT mice. Consistently, the density of CD31-positive microvessels from metastasized foci after intrasplenic injection of SL4 cells was significantly smaller in Cat $\mathrm{S}^{-/-}$mice than those in WT mice (Additional file 1: Figure S1B). Therefore, the absence of stroma-derived Cat S inhibited tumor growth, metastasis and angiogenesis, supporting a tumor-promoting role of infiltrating leukocytes expressing Cat $\mathrm{S}$.

\section{Cat S deficiency inhibits transition toward the M2 phenotype in TAMs}

To further analyze the cellular origin of Cat $\mathrm{S}$ expression in tumor-infiltrating leukocytes, tumor sections were immunostained with the macrophage marker Mac-2 to assess macrophage infiltration into the tumor stroma. Immunohistochemical staining demonstrated that the expression of Mac-2 in hepatic metastatic tumors did not significantly differ between WT and Cat $\mathrm{S}^{-1-}$ mice (Figure 3A and B). Moreover, Cat $\mathrm{S}$ was highly expressed in macrophages, while Cat $\mathrm{S}^{-1-}$ macrophages showed no Cat $\mathrm{S}$ expression (Figure 3C). Furthermore, we did not observe a significant difference in F4/80-positive cells between WT and Cat $\mathrm{S}^{-/-}$ mice in hepatic metastatic tumors (Figure 3C).

TAMs are prominent components of leukocyte infiltrates and are frequently polarized to an M2 phenotype, which is characterized by tumor-promoting properties [4,6,33]. To further establish whether Cat $\mathrm{S}$ is necessary for M2 macrophage polarization in the tumor microenvironment, we assessed the cell surface expression of CD206, a marker of M2 macrophage. Immunofluorescence analysis revealed that double-positive F4/80 and CD206 viable macrophages were abundant in the tumors of WT mice but not in Cat $\mathrm{S}^{-/-}$mice (Figure 3D). We further analyzed the macrophage phenotypes in tumors using flow cytometric analysis. As shown in Figure 3E, the population of macrophages $\left(\mathrm{CD} 45^{+} \mathrm{F} 4 / 80^{+}\right.$cells) in hepatic metastatic tumors of WT mice was predominantly CD206-positive, while the proportion of $\mathrm{CD} 45^{+} \mathrm{F} 4 / 80^{+} \mathrm{CD} 206^{+}$cells was significantly reduced in the tumors of Cat $\mathrm{S}^{-/-}$mice $(13.99 \pm 1.45 \%$ versus $37.09 \pm 2.81 \%$ for $\mathrm{CD} 45^{+} \mathrm{F} 4 / 80^{+} \mathrm{CD} 206^{+}$cells, $\left.P<0.01\right)$ (Figure $3 \mathrm{E}$ ). These results suggest that $\mathrm{Cat} S$ may drive macrophages toward the M2 phenotype in the tumor microenvironment.

\section{Cat S deficiency induces autophagosome accumulation in TAMs}

Previous studies showed that autophagy is critical for malignant progression of tumors, serving to both reduce oxidative stress and provide key intermediates to sustain cell metabolism $[34,35]$. We recently reported that Cat S deficiency results in abnormal accumulation of autophagosomes in macrophages with angiotensin II treatment [31]. Therefore, we further analyzed whether the role of Cat $S$ in the regulation of TAMs polarization was linked to autophagy. On immunofluorescence staining, we observed colocalization of the autophagosomal marker LC3 and macrophage marker F4/80 in the hepatic metastatic tumors of WT mice, and this effect was markedly increased in Cat $^{-1-}$ mice (Figure 4A).

To examine the expression of Cat $S$ in macrophages, double-color immunofluorescence analysis was performed in bone marrow-derived macrophages. As shown in Additional file 2: Figure S2A, Cat S was expressed in F4/80-positive macrophages. Importantly, the level of Cat S expression in WT macrophages was markedly elevated after coculture with SL4 cells under serumstarved conditions. Western blotting demonstrated that SL4 cells significantly increased the level of Cat S protein in WT macrophages under serum-starved conditions (Additional file 2: Figure S2B). To further confirm the role of Cat $\mathrm{S}$ in vitro, confocal fluorescence microscopy showed that SL4 cells stimulated LC3 protein clustering in WT macrophages under serum-starved conditions, which was further enhanced in Cat $\mathrm{S}^{-/-}$macrophages (Figure 4B).

To investigate whether autophagic structures were increased in Cat $\mathrm{S}^{-1-}$ macrophages in the tumor microenvironment, we detected autophagic vesicles by transmission electron microscopy (TEM). Autophagic vesicles are hallmarked by unique double membranes and by the presence of engulfed cytosolic content: features that allow them to be easily detected by TEM. Although WT or Cat $\mathrm{S}^{-/-}$macrophages contained very few autophagic vesicles and did not differ in the formation of autophagosomes at baseline, SL4 cells induced cytosolic autophagic vacuole formation in serum-starved WT macrophages (Figure 4C). Especially, many large vesicles containing engulfed and digested membrane and organelle structures were observed in Cat $\mathrm{S}^{-/-}$macrophages with SL4 cells coculture (Figure 4C).

The formation of phagophores and autophagosomes is associated with lipidation of LC3-I with phosphatidylethanolamine to produce the membrane-associated LC3-II 


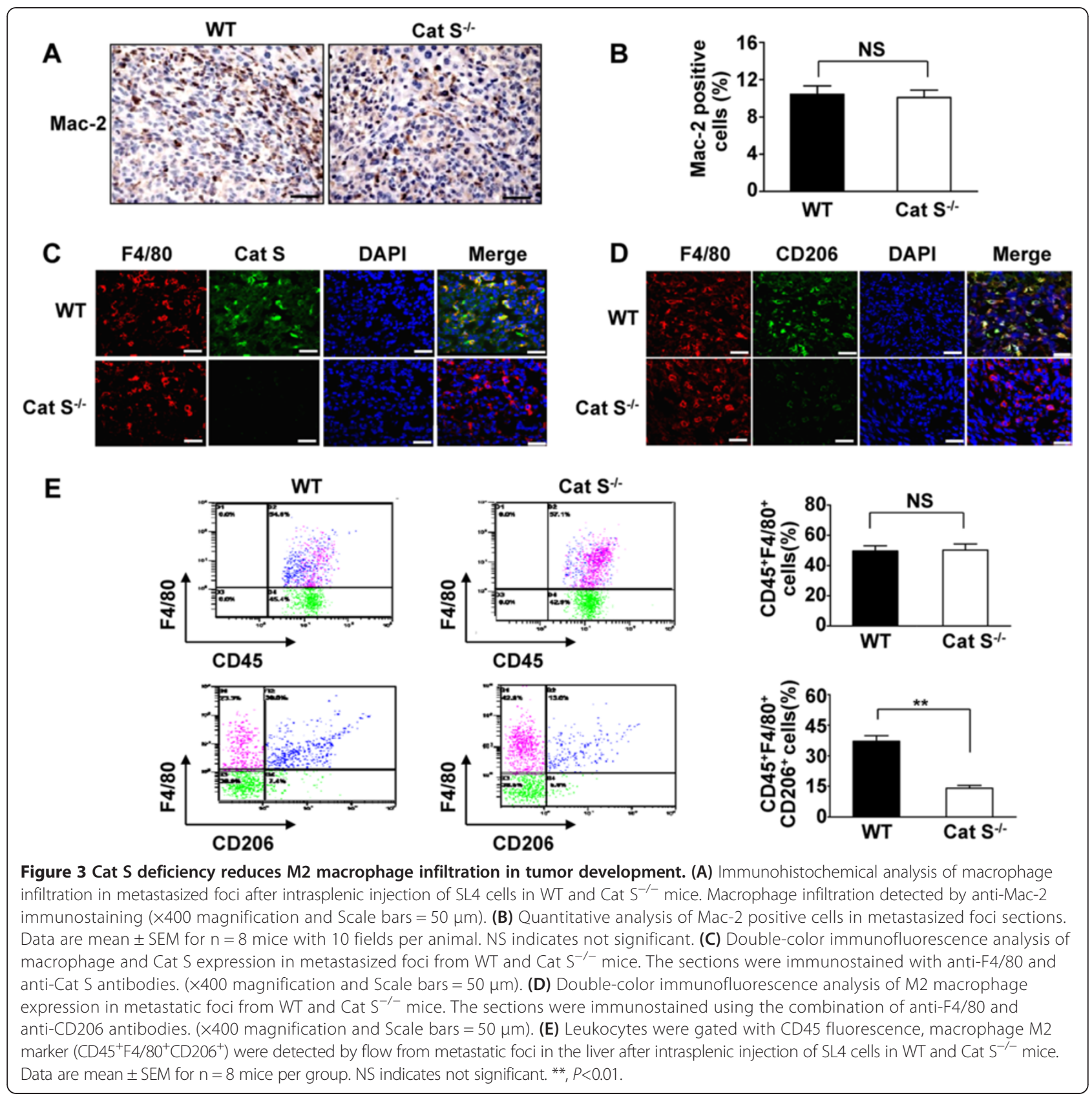

protein [36]. We further measured autophagosomeassociated LC3 (LC3 II) and free cytosolic LC3 (LC3-I) by western blotting. As shown in Figure 4D, in control, the LC3-II formation was in low level either WT or Cat $\mathrm{S}^{-1-}$ macrophages, no difference between the two groups. Consistent with autophagic vesicle accumulation, SL4 cells induced LC3-II formation in WT macrophages, which was even further enhanced in Cat $\mathrm{S}^{-/-}$macrophages (Figure 4D). Our results suggest that Cat S deficiency may induce the accumulation of autophagosomes in macrophages with the tumor microenvironment.
Cat $S$ is required for autophagic flux of TAMs

The flux rate of autophagy can be controlled by several steps: 1) the enclosure of cytoplasmic components by phagophores to form autophagosomes; 2) the fusion of autophagosomes with lysosomes to form autophagolysosomes; 3) the dissolution of autophagolysosomes [11,15]. To investigate whether Cat $S$ is involved in the initiation and progression of autophagy, autophagic flux was assessed using colocalization analysis of the tandem fluorescent mCherry-GFP-LC3 expression vector. In autophagosomes, both GFP and mCherry fluorescence 


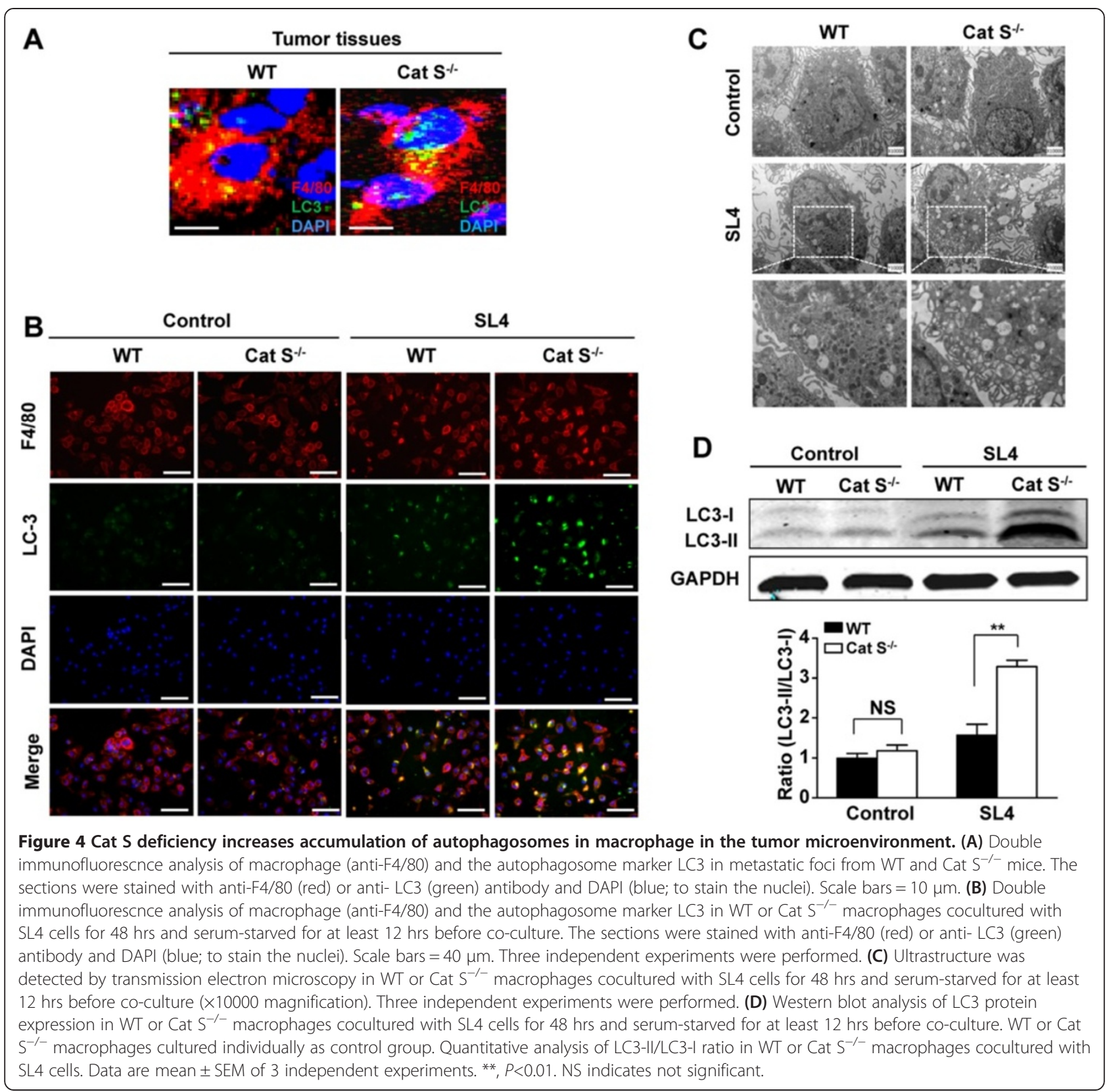

is colocalized. In autophagolysosomes, the green fluorescence in the mCherry-GFP-LC3 fusion protein weakens and eventually disappears under acidic $\mathrm{pH}$, making it a fluorescent sensor to simultaneously analyze autophagosomes and autophagolysosomes [37]. Progression from autophagosomes to autophagolysosomes, therefore, presents as less colocalization and stronger red fluorescence.

In Cat $\mathrm{S}^{-/-}$or WT macrophages, the lower basal level of autophagy presented as low-intensity fluorescence and was poorly localized (Figure 5A). We found that mCherry-GFP-LC3 expression was detected as predominantly red and yellow spots rarely observed in WT macrophages cocultured with SL4 cells, suggesting that the expression is mainly associated with autophagolysosomes (Figure 5A). In contrast, in Cat $\mathrm{S}^{-/-}$macrophages cocultured with SL4 cells, yellow speckles with a higher level of GFP and mCherry fluorescence and their colocalization were significantly observed compared with those in WT macrophages (Figure 5A, right panels). The inhibition of fusion of autophagosomes with lysosomes resulted in the accumulation of mCherry-GFP-LC3 on autophagosomes; therefore, the number of GFP-LC3 puncta per cell was substantially higher in Cat $\mathrm{S}^{-/-}$macrophages than in WT macrophages cocultured with SL4 cells, with no difference between the two cell types at baseline (Figure 5B). Our results indicated that Cat $\mathrm{S}$ deficiency inhibited 


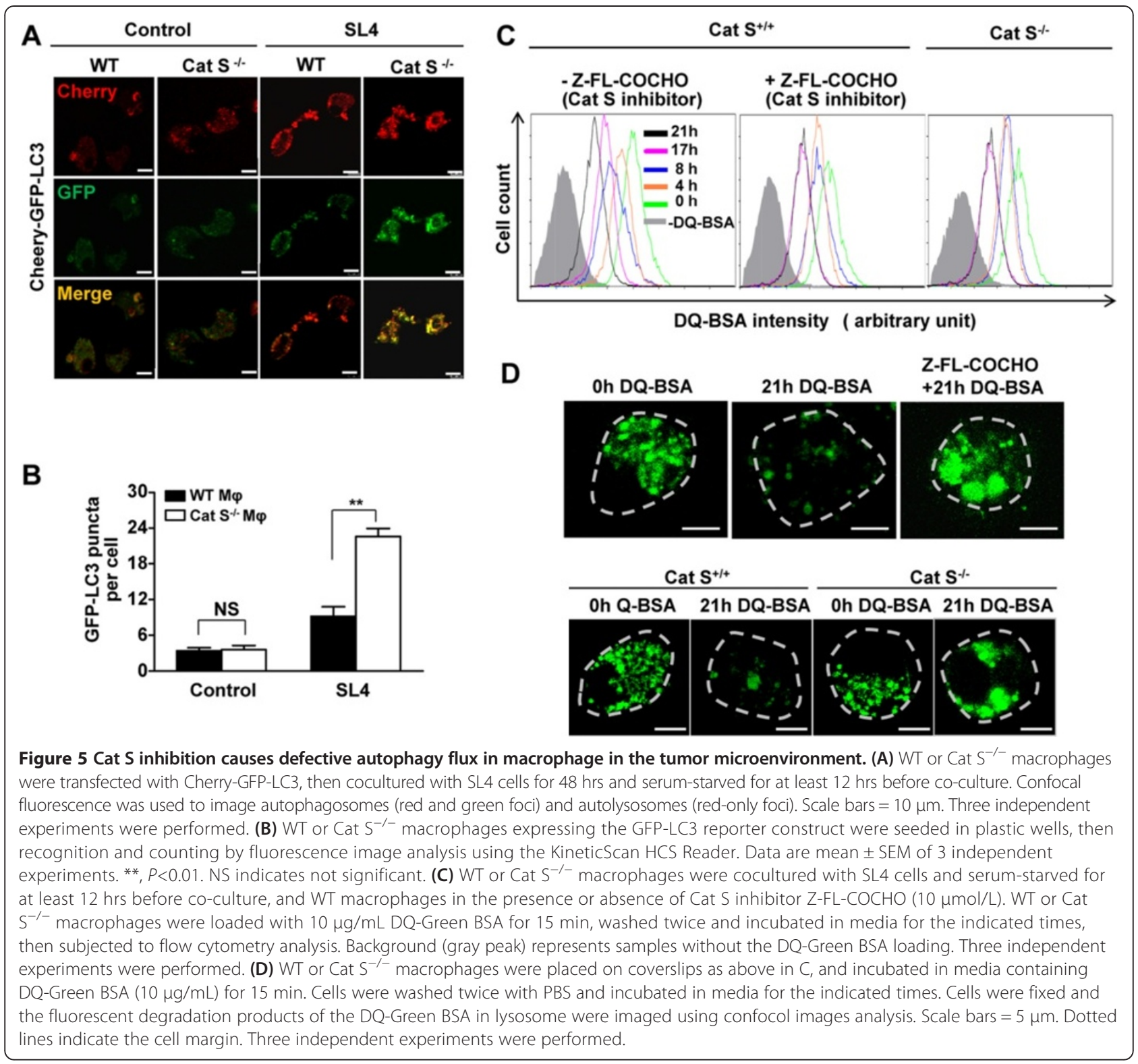

autophagosome degradation to slow down the autophagy flux rate in TAMs.

An increase in autophagy should coincide with enhanced lysosomal activity and general proteolysis. We utilized the self-quenched reporter substrate, $\mathrm{DQ}^{\mathrm{TM}}$ Green BSA (DQ-BSA) to measure of the cellular proteolytic/lysosomal activity. DQ-BSA-green is a bovine serum albumin (BSA) labeled with a self-quenching fluorescent dye. After DQBSA-green is delivered to lysosomes via endocytosis, it is hydrolyzed into single dye-labeled peptides by lysosomal proteases, thereby relieving self-quenching, and the green fluorescence can subsequently be monitored by flow cytometry [38]. Thus, DQ-BSA is useful for detecting intracellular proteolytic activity as a measure of a functional lysosome. To further examined whether Cat $\mathrm{S}$ deficiency could prevent autophagy flux, DQ Green BSA was preloaded and dequenched in macrophages, and the rate of disappearance of dequenched DQ-BSA was monitored. As shown in Figure 5C, left panel, fluorescent signals were decreased by approximately $90 \%$ over the time period of 21 hrs in WT macrophages cocultured with SL4 cells. However, in the presence of the Cat S inhibitor Z-FL-COCHO, fluorescence peaks shifted to the left was obviously inhibited, demonstrating that the flux of dequenched DQ-BSA was significantly delayed (Figure 5C, middle panel). Similarly, we observed the loss of the fluorescent signals was markedly blocked in Cat $\mathrm{S}^{-1-}$ macrophages compared with that in WT macrophages cocultured with SL4 cells, indicating that Cat $\mathrm{S}$ deficiency attenuated the DQ-BSA degradation rate 
(Figure 5C, right panel). Additionally, WT macrophages treated with the Cat $\mathrm{S}$ inhibitor or Cat $\mathrm{S}^{-/-}$macrophages showed enlarged dequenched DQ-BSA-containing vesicles (Figure 5D, top and bottom panels). These results consistently suggested that $\mathrm{Cat} S$ is required for clearing dequenched DQ-BSA or flux of amphisomes.

\section{Cat S-mediated autophagy contributes to M2 phenotype polarization of TAMs}

To further confirm that Cat S-mediated autophagy plays an important role in macrophage polarization in the tumor microenvironment, we treated WT or Cat $\mathrm{S}^{-/-}$ macrophages with the autophagy inhibitor chloroquine (CQ) prior to coculture with SL4 cells, and assayed the expression of M2-type genes (arginase-1, Arg-1; found in inflammatory zone protein, FIZZ1 and interleukin-10, IL-10) and M1-type genes (inducible nitric oxide synthase, iNOS; interleukin-1 $\beta$, IL-1 $\beta$ and tumor necrosis factor$\alpha$, TNF- $\alpha$ ). As shown in Figure 6, coculture of SL4 cells markedly upregulated the mRNA levels of Arg-1, FIZZ1 and IL-10 (M2-specific genes) in WT macrophages, whereas CQ significantly inhibited the expression of Arg-1, FIZZ1 and IL-10 in WT macrophages cocultured with SL4 cells. Moreover, coculture of SL4 cell induced a significant increase in expression of Arg-1, FIZZ1 and IL-10 in WT macrophages compared to that of Cat $\mathrm{S}^{-/-}$macrophages (Figure 6). Importantly, CQ treatment did not alter the expression of Arg-1, FIZZ1 or IL-10 in Cat $\mathrm{S}^{-/-}$macrophages cocultured with SL4 cells (Figure 6, top panels). In addition, WT macrophages treated with CQ expressed higher levels of iNOS, displaying an M1-skewed profile when cocultured with SL4 cells (Figure 6). Cat $\mathrm{S}^{-1-}$ macrophages also showed a marked increase in the expression of iNOS compared with that in WT macrophages following coculture with SL4 cells, and this profile was not further altered in Cat $\mathrm{S}^{-1-}$ macrophages with CQ treatment (Figure 6, bottom panels). Taken together, Cat S-mediated autophagy promotes the polarization of TAMs toward an M2-like phenotype.

\section{Discussion}

TAMs represent a dominant myeloid population in many solid tumors, and their accumulation correlates with poor prognosis [7,33]. TAMs-secreted cathepsin protease activity has been implicated in the pathogenesis of cancer [30], yet the mechanisms by it promotes tumor development are incompletely understood. In this study, we identified Cat $S$ as the most abundantly expressed cysteine protease of the cathepsins family in tumor-infiltrating macrophages, and its level was associated with poor prognosis in human

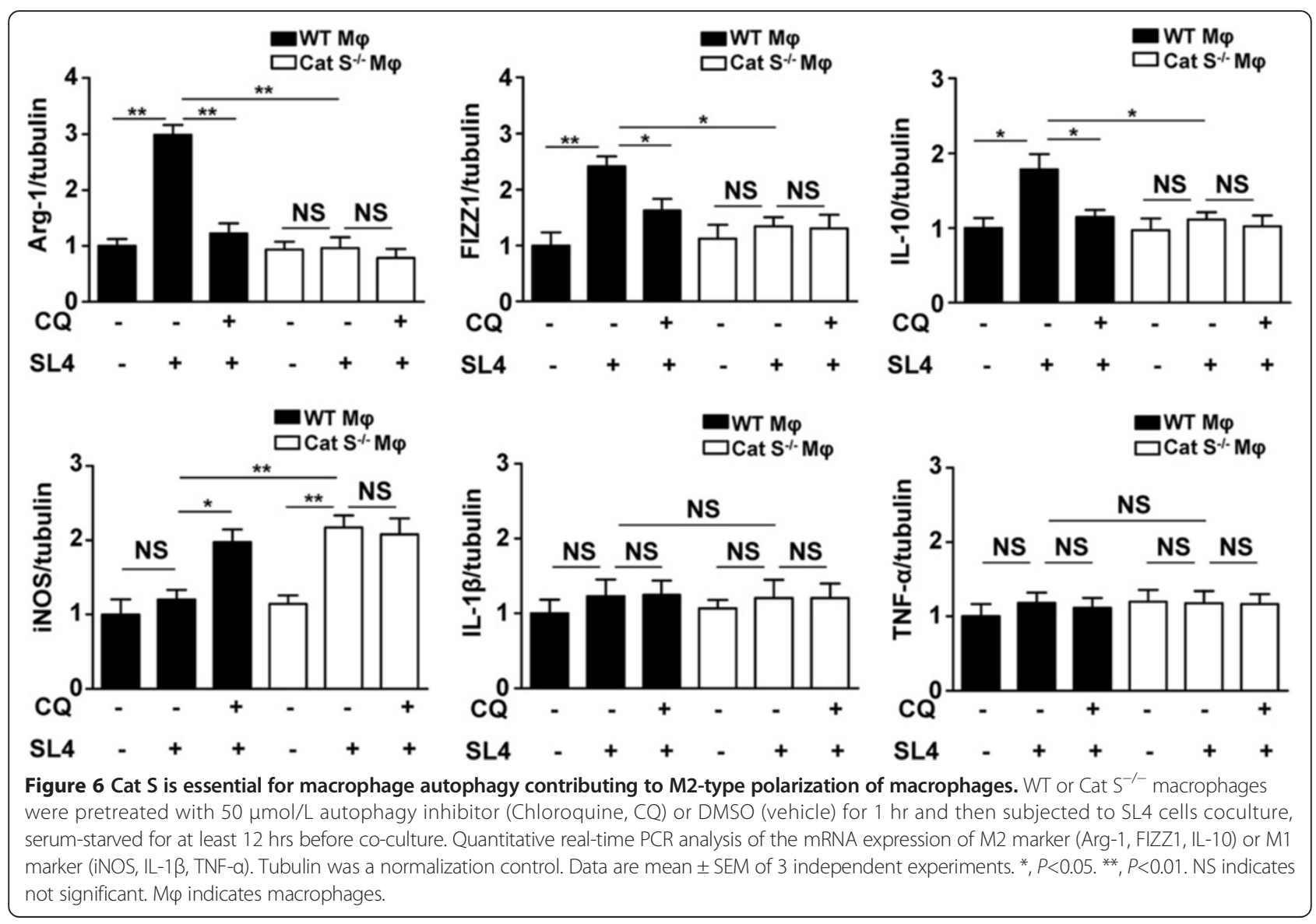


colon carcinoma. We showed that Cat $\mathrm{S}$ functions as a potentiator of tumor development through maintaining macrophage cellular homeostasis and mediating the switch to an M2 phenotype in TAMs. Moreover, we gained further insights into the potential mechanisms underlying Cat $S$ as a critical mediator of autophagic flux, the cancer-promoting function of TAMs.

Clinical studies have shown that Cat $S$ is expressed in $95 \%$ of cases of primary colorectal tumors and their related metastatic tissue, with significantly higher expression in tumors compared with matched normal colonic mucosa [39]. In vitro findings demonstrated that specific inhibition of Cat S by an antibody, Fsn0503, could attenuate colorectal cancer cell invasion [25]. Furthermore, these effects were confirmed in a murine model of sporadic pancreatic carcinogenesis (RIP1-Tag2), in which the genetic ablation of Cat S severally inhibited tumorigenesis through attenuation of tumor invasion and angiogenesis [23]. However, the mechanism by which stroma-derived Cat $\mathrm{S}$ promotes tumor development remains unclear. Therefore, in present investigation, we aimed to investigate the role and the mechanism of Cat $\mathrm{S}$ in regulating tumor microenvironment. In this study, we observed that colon carcinoma patients at more advanced clinical stages, those with lymph node or liver involvement, or those who have had recurrence within 3 years displayed markedly higher Cat $\mathrm{S}$ expression levels (Figure 1B). These results indicate a potential of Cat $\mathrm{S}$ as an independent or supplementary biomarker in the prediction of tumor prognosis. Furthermore, we found development of metastasized tumors in the liver and tumor growth of $\mathrm{PancO}_{2}$ cells were greatly inhibited in Cat $\mathrm{S}^{-1-}$ mice compared to WT littermates (Figure 2), suggesting a critical role of TAMs Cat $\mathrm{S}$ expression in contributing to tumor development.

Besides of colon cancer tissues, previous studies have shown that significantly high levels of Cat $\mathrm{S}$ have been reported in a range of tumors including glioma [28], astrocytoma [24], lung cancer [40], prostate cancer [41], hepatocellular [27], and pancreatic carcinomas [42], revealing a possible role for this enzyme in tumour growth and progression. Cat $\mathrm{S}$ activity is also present in astrocytoma cells in vitro and the extracellular levels of activity were highest in cultures derived from grade IV tumors [24]. Furthermore, tumor cells such as breast cancer cells, pancreatic or colon cancer cells, express functional Cat $\mathrm{S}$ which has been proposed to involve in invasiveness of cancer cells $[43,44]$.

In addition to the production of Cat $\mathrm{S}$ by the tumor cells, further analysis confirmed that TAMs produced the protease as previously shown in the mouse models for pancreatic cancer and breast cancer $[23,30,45,46]$. Several studies have shown that expression of Cat $\mathrm{S}$ by tumour-infiltrating macrophages, could be an important contributor during prostate cancer progression [41]. Recent studies have demonstrated that both tumor cells and tumor-associated macrophages can produce Cat $\mathrm{S}$ within the microenvironment to promote neovascularization and tumor growth [47]. In the present study, the data showed that Cat S deficiency impaired tumor angiogenesis in metastatic foci. Consistent with our results, Cat $\mathrm{S}$ null mice exhibited impaired endothelial microvessel development, suggesting a key role for this protease in angiogenesis [25]. Similarly, Cat S is markedly up-regulated by endothelial cells during tumour angiogenesis $[27,42]$ and importantly, in a murine pancreatic islet carcinoma model (RIP1-Tag2), Cat S knockout mice have been shown to a significant reduction in tumour-associated angiogenic switching and neovascularisation [23]. Furthermore, the Cat S inhibitory antibody, Fsn0503, blocks extracellular proteolysis, inhibiting endothelial invasion and tube formation into developing tumors [48]. Taken together, reduced angiogenesis in Cat $\mathrm{S}$ deficient mice could also partially contribute to impaired tumor develpoment.

Lysosomal cysteine proteinases are known to mediate intracellular protein turnover, regulating the half-life of proteins critical for normal cell function. Cat $S$ is restricted to lymphatic tissues and cells such as macrophages, indicating more specific roles in cell physiology $[40,49,50]$. Given that macrophages are major participants in host inflammatory responses, dysregulation of macrophage function may lead to diseases including autoimmune disease and cancer [3]. In the present study, we provide both in vitro and in vivo evidence that $C$ at $\mathrm{S}$ is required for $\mathrm{M} 2$ polarization and cancer-promoting functions of macrophages in the tumor microenvironment.

Autophagy is upregulated in response to stress, including growth factor and nutrient limitation, energy depletion, and hypoxia [12,51]. As a cell-refreshing and metabolismsupporting pathway, autophagy is required for the normal operation of cellular and organismal physiology. Therefore, through selective autophagy, the central metabolism may be supported by different substrates to restore metabolic and energy homeostasis, redox balance, and biomass production. Thus, autophagy can enable stress adaptation, maintenance of cellular fitness, and survival under different stress conditions [14]. In advanced cancers, autophagy can promote tumor progression by providing nutrients during starvation $[14,16,52]$. These findings suggest that autophagy inhibition, rather than stimulation, might be beneficial in the treatment of advanced cancer. Given the known importance of TAMs in cancer development and progression, the response of tumors to autophagy inhibition may also involve the immune system. One of the most important functions of autophagy is to maintain cellular energy under conditions of nutrient deprivation and other forms of stress [15]. The present study established a novel role of macrophage-secreted Cat $S$ in the proper execution of autophagy. We showed that Cat $\mathrm{S}$ was required for efficient autophagic flux, based on the 
facts that Cat S-deficient macrophages showed more accumulation of autophagic vacuole-like structures and multivesicles than WT macrophages by serum starvation during SL4 cells coculture (Figure 4C). Moreover, significant higher levels of LC3-II were detected in Cat S-deficient macrophages than WT macrophages (Figure 4D). Autophagy is a vacuolar lysosomal degradation pathway for long-lived proteins and damaged organelles that are critical for maintaining cell function under stress conditions. During the late stage of autophagy, autophagosomes are presented to lysosomes to degrade damaged organelles (i.e., mitochondria) [12,53]. In the present study, Cat $\mathrm{S}$ deficiency increased autophagosome formation in macrophages in the tumor microenvironment (Figure 5A). Furthermore, we also showed that Cat $\mathrm{S}$ was required for the fusion processes of autophagosomes and lysosomes, as there were significantly defects in the dequenching process of DQ-BSA in Cat S inhibitor-treated macrophages and Cat S-deficient macrophages cocultured with SL4 cells (Figure 5C and D). These results suggest that the fusion of the autophagic vacuole with the lysosome and the subsequent degradation of its content by Cat $S$ are crucial for proper execution of autophagy in TAMs.

Macrophages are highly heterogeneous cells that can be activated to a functional status between M1 and M2 phenotypes in response to environmental signals. In a simplified view, macrophages activated by LPS and IFN- $\gamma$ are referred to as M1 macrophages, which are capable of killing pathogens and tumor cells $[4,5]$. Macrophages activated by IL-4, IL-13, and IL-10 are referred to as M2 macrophages, which can suppress inflammation, induce angiogenesis, promote tissue repair, and enhance tumor growth $[4,5]$. We demonstrated that the autophagy inhibitor CQ impairs the transition toward the M2 phenotype in macrophages within the tumor microenvironment. Importantly, lack of Cat S and CQ treatment did not produce additive suppression of M2 macrophage transition within the tumor microenvironment, further highlighting the biological necessity of Cat S-mediated autophagy in mediating M2-type polarization of TAMs.

Cat $\mathrm{S}$, a cysteine protease of the papain family, is expressed in lysosomal/endosomal compartments of antigen-presenting cells, such as B cells, macrophages and dendritic cells (DCs) [54]. Inside the B cells and DCs, Cat S is the single enzyme during the assembly of the MHC class II- $\alpha$ and II- $\beta$ chains with the antigenic peptide in the lysosomal/endosomal compartments [55]. This process contributes to antigen-induced adaptive immunity. Moreover, Cat $\mathrm{S}$ has been shown to dominate autoantigen processing in human thymic dendritic cells, which would be an important factor that influences selection of autoreactive $\mathrm{T}$ cells [56]. These findings suggest the role of Cat $S$ in other immune cells could potentially also invovle in changes of our observed tumor development in this study.

\section{Conclusions}

In summary, the activity of Cat S plays an important role in macrophage autophagy in the tumor microenvironment, leading to regulation of the M2 phenotype of TAMs. The results of the present study provide a strategy to selectively target inflammatory cells in combination with autophagy, an approach that could have significant therapeutic potential. Thus, controlling the activity of Cat $\mathrm{S}$ involved in autophagy, by developing drugs that target this process, can be considered as a future antitumor strategy.

\section{Materials and methods \\ Antibodies and reagents}

The antibodies for CD68, Ki-67, CD31, Mac-2, GAPDH, and IgG were from Santa Cruz Biotechnology (Santa Cruz, CA); the antibodies for Cat S, F4/80 and LC-3 was from Abcam (Cambridge, MA); and ChemMate TM EnVision System/DAB Detection Kits were from Dako (Glostrup, Denmark). Antibodies for PerCP/Cy5.5conjugated CD45.2, phycoerythrin (PE)-conjugated F4/80, fluorescein isothiocyanate (FITC)-conjugated CD206 and isotype control were from Biolegend (San Diego, CA). Cat S inhibitor, Z-FL-COCHO was purchased from Calbiochem (San Diego, CA). Autophagy inhibitor, Chloroquine were purchased from Sigma (St. Louis, MO).

\section{Animals}

The Cat $\mathrm{S}^{-1-}$ mouse strain was backcrossed onto the genetic background of C57BL/6 for more than 10 generations. Mice were 8-12 weeks old at the beginning of the experiments, matched for age and sex with wild-type (WT) mice, and kept under specific pathogen-free (SPF) conditions at the Beijing Anzhen Hospital affiliated to the Capital University of Medical Science, China. All animals received humane care in compliance with the Animal Management Rule of the Ministry of Health, People's Republic of China (documentation no. 55, 2001) and the Care and Use of Laboratory Animals published by the US National Institutes of Health (NIH Publication No. 85-23, revised 1996) and approved by the Institutional Animal Care and Use and Committee of the Capital University of Medical Science (Beijing, China).

\section{Tumor model}

PancO2 [57] and SL4 [32] cells are pancreatic and colon cancer cells, respectively, derived from C57BL/6 mice on the same background as the Cat $\mathrm{S}^{-/-}$mice and WT control mice. PancO2 and SL4 cells were maintained in DMEM/ F12 culture medium, supplemented with $10 \%$ FBS in a humidified $37^{\circ} \mathrm{C}$ incubator under $5 \% \mathrm{CO}_{2}$.

For in vivo subcutaneous tumor model, PancO2 cells were harvested and single-cell suspensions of $1.0 \times 10^{6}$ cells in $200 \mu \mathrm{l}$ medium were injected subcutaneously into the right flank. Mice were sacrificed 28 days after 
subcutaneous injection. Tumor volume was measured with a caliper using the formula: $V=\pi \times\left[d^{2} \times D\right] / 6$, where $d$ is the minor tumor axis and $\mathrm{D}$ is the major tumor axis. For in vivo hepatic metastasis model, after anaesthetizing mice, a transverse incision in the left flank was made, exposing the spleen, $1.0 \times 10^{6} \mathrm{SL} 4$ tumor cells in $100 \mu \mathrm{DMEM} / \mathrm{F} 12$ medium were intrasplenically injected with use of a 26-gauge needle. 14 days after inoculation, mice were sacrificed, and the tissues were processed as described below. The spleen and liver were removed, wet spleen and liver weights were measured, and the incidence of liver metastasis was examined.

\section{Human colon carcinoma specimens}

The specimens from 30 cases of human colon carcinoma tissue/adjacent normal colon tissues and the clinicopathologic data were obtained from the Second Affiliated Hospital to Nanchang University gastrointestinal tumor bank. The specimens were isolated at the time of surgery, formalin-fixed and paraffin-embedded, and stained with hematoxylin and eosin, then examined by 2 experienced pathologists. The clinicopathologic stage was determined according to the TNM classification system of the International Union against Cancer. RNA was extracted for fresh tissue specimens (from 15 colon carcinoma specimens and 15 adjacent normal colon tissues) with identifiable tumor in the tissue specimen. Human specimens use for research had been approved by the Second Affiliated Hospital to Nanchang University Research Ethics Committee.

\section{Histology and immunohistochemistry}

Specimens were fixed for $24 \mathrm{hrs}$ with $10 \%$ buffered formalin before embedding in paraffin. Serial sections of $5 \mu \mathrm{m}$ thick were obtained for histologic analysis. Hematoxylin\&eosin (HE) staining involved standard procedures.

For immunohistochemistry, sections were incubated with the primary antibodies for Cat S (1:200), CD68 (1:200), Ki-67 (1:200), CD31 (1:200), Mac-2 (1:200), then incubated with the Dako ChemMateTM EnVision System (Dako, Glostrup, Denmark) for $30 \mathrm{~min}$. Staining was visualized with use of diaminobenzidine and counterstaining with hematoxylin. Negative controls were omission of the primary antibody, non-immune IgG or secondary antibody only; in all cases, negative controls showed insignificant staining. The expressions of Cat S, CD68, Ki-67, CD31, Mac-2 were calculated as proportion of positive area to total tissue area for all measurements of the section.

For double immunofluorescence, $7 \mu \mathrm{m}$ frozen tissue sections were permeabilized and blocked with $0.1 \%$ Triton $\mathrm{X}-100,0.2 \%$ bovine serum albumin, and $5 \%$ normal donkey serum in PBS, then incubated with the primary antibodies F4/80 (1:100), LC-3 (1:200) and Cat S (1:200) overnight at $4^{\circ} \mathrm{C}$, then FITC or TRITC-conjugated secondary antibody
(Jackson Immuno Research Laboratories, West Grove, PA, USA) at $4^{\circ} \mathrm{C}$ for $1 \mathrm{hr}$ in the dark, and coverslipped with DAPI-containing mounting medium.

\section{RNA analysis}

Total RNA were extracted by use of TRIZOL (Invitrogen, Carlsbad, CA, US). For reverse transcription, $1 \mu \mathrm{g}$ of total RNA was used to generate first strand CDNA with OligodT primer. Real-time PCR was performed using the SYBR Green Mix (Bio-Rad) on the CFX96 Real-time System (Bio-Rad). The Table 1 shows the primer used.

\section{Western blotting}

Protein extracts were diluted with loading buffer and separated by electrophoresis on $8 \%-10 \%$ SDS-polyacrylamide gels before transfer to nitrocellulose membranes (Bio-Rad). The membranes were blocked in Odyssey blocking buffer (LI-COR Bioscience, Lincoln, NE) at room temperature for $1 \mathrm{hr}$, then incubated at $4^{\circ} \mathrm{C}$ overnight with primary antibodies: Cat S (1:1000), LC-3 (1:1000), GAPDH (1:3000). The membranes were washed 3 times in TBST and incubated with fluorescent secondary antibodies (Alexa Fluor 680 or IRDye 800, Rockland Immunochemicals, Gilbertsville, PA, US) for $1 \mathrm{hr}$ at room temperature at 1:5000, blots were analyzed with the Odyssey infrared imaging system and Odyssey software.

\section{Flow Cytometry}

The content of inflammatory cells was quantified by flow cytometry as described [58]. Briefly, tumor tissues were cut into multiple small cubes and digested in an enzyme mixture for 45 mins at $37^{\circ} \mathrm{C}$. The cell suspension was centrifuged and pre-incubated with Fc- $\gamma$ block antibody (anti-mouse CD16/32; Pharmingen, San Diego, CA, USA) to prevent nonspecific binding. Cell staining involved different combinations of fluorochrome-coupled antibodies to $\mathrm{CD} 45, \mathrm{~F} 4 / 80$, $\mathrm{CD} 206$ for 30 mins at $4^{\circ} \mathrm{C}$ in the dark. Fluorescence data were collected by use of an EPICS XL flow cytometer (Beckman Coulter) and analyzed by use of Cellquest (Beckman). Fluorescence minus one (FMO) controls were included to determine the level of nonspecific staining and auto-fluorescence associated with subsets of cells in each fluorescence channel.

\section{Co-cultures of bone marrow-derived macrophages (BMDMs) and tumor cells}

Co-culture systems were established by using transwell inserts $(0.4 \mathrm{~mm}$ pore, polycarbonate membrane; Costar, Cambridge, USA) and transferred to 6-well culture plates. Bone marrow-derived macrophages (BMDMs) were isolated from tibias and femurs of 8-week-old wild-type C57BL/6 or Cat $\mathrm{S}^{-1-}$ mice as previously described [58]. SL4 cell suspensions $\left(1 \mathrm{ml}, 1 \times 10^{6}\right.$ cells) were loaded in the upper inserts, and WT or Cat $\mathrm{S}^{-/-}$BMDMs suspensions 
Table 1 Primers used for qRT-PCR

\begin{tabular}{|c|c|c|}
\hline Primer & Forward & Reverse \\
\hline Human cathepsin B & 5'gtttgcattgctggtcagga3' & 5'tggcaggacagtggaatgat3' \\
\hline Human cathepsin D & 5'gcgagtacatgatcccctgt3' & 5'ctctggggacagcttgtagc3' \\
\hline Human cathepsin F & 5' tggcaacaagatgaagcaag3' & 5'ttttgtgacageccccttac3' \\
\hline Human cathepsin $\mathrm{H}$ & 5'actggctgttgggtatggag3' & $5^{\prime}$ aggecacacatgttctttcc $3^{\prime}$ \\
\hline Human cathepsin $\mathrm{K}$ & 5'ccgcagtaatgacacccttt3' & $5^{\prime}$ gcacccacagagctaaaagc $3^{\prime}$ \\
\hline Human cathepsin L & 5'acagtggaccaagtggaagg3' & $5^{\prime} \mathrm{cttctcc}$ acactgctctcc $3^{\prime}$ \\
\hline Human cathepsin S & 5'tcatacgatctgggcatgaa3' & 5'aggttctgggcactgagaga3' \\
\hline Human cathepsin Z & 5'aagggggtaatgacctgtcc3' & 5'ttcattgcatgtcccacatt3' \\
\hline Human GAPDH & 5'acagtcagccgcatcttctt3' & 5'acgaccaaatccgttgactc3' \\
\hline Mouse Arg-1 & 5'aaagctggtctgctggaaaa3' & $5^{\prime}$ acagaccgtgggttcttcac3' \\
\hline Mouse FIZZ1 & 5'ttgcaactgcctgtgcttac3' & 5'ctgggttctccacctcttca3' \\
\hline Mouse IL-10 & 5'ccaagccttatcggaaatga3' & 5'ttttcacaggggagaaatcg3' \\
\hline Mouse iNOS & 5'gggctgtcacggagatca3' & 5'ccatgatggtcacattctgc3' \\
\hline Mouse IL-1 $\beta$ & 5'gcccatcctctgtgactcat3' & 5'aggccacaggtattttgtcg3' \\
\hline Mouse TNF-a & 5'tcttctcattcctgcttgtgg $3^{\prime}$ & 5'ggtctgggccatagaactga3' \\
\hline Mouse Tubulin & 5'tctaacccgttgctatcatgc3' & 5'gccatgttccaggcagtag3' \\
\hline
\end{tabular}

GAPDH, glyceraldehyde 3-phosphate dehydrogenase; Arg-1, arginase-1; FIZZ1, found in inflammatory zone 1; IL-10, interleukin 10; iNOS, inducible NO synthase; IL-1 $\beta$, interleukin $1 \beta$; TNF-a, tumor necrosis factor-alpha.

( $3 \mathrm{ml}, 3 \times 10^{6}$ cells) were put into the lower compartment of the culture well for $48 \mathrm{hrs}$ and serum-starved for at least 12 hrs before co-culture. Serum-free DMEM/F12 without SL4 cell inserts was used as a control in the lower compartment of well. BMDMs were preincubated for $1 \mathrm{hr}$ with $10 \mu \mathrm{mol} / \mathrm{L}$ Cat S inhibitor Z-FL-COCHO or $50 \mu \mathrm{mol} / \mathrm{L}$ autophagy inhibitor Chloroquine, immediately after SL4 cells plating and controls received equivalent dilution with DMSO vehicle alone.

\section{Transmission electron microscopy}

For transmission electron microscopy (TEM), cells were fixed in $2 \%$ glutaraldehyde in $0.1 \mathrm{M}$ sodium cacodylate buffer ( $\mathrm{pH} 7.0$ ) for $2 \mathrm{hrs}$, post-fixed in $2 \%$ osmium tetroxide for $2 \mathrm{hrs}$ and then rinsed with $0.1 \mathrm{M}$ cacodylate buffer. Cells were enrobed in 5\% Noble Agar and washed with distilled water 5 times, further fixing with $2 \%$ uranyl acetate for 2 hrs, followed by dehydration in 50\% (15 $\mathrm{min}$ ), 70\% (16 h), 85\% (15 $\mathrm{min}), 95 \%$ (15 $\mathrm{min}$ ), and 2 changes of $100 \%$ ethanol each $15 \mathrm{~min}$. They were then cleared by 2 changes of propylene oxide, each $15 \mathrm{~min}$, and infiltrated with epon resin:propylene oxide (1:1) for $3 \mathrm{hrs,} \mathrm{epon} \mathrm{resin:propylene}$ oxide (3:1) for $16 \mathrm{hrs}$, and 2 changes with pure epon resin for total $6 \mathrm{hrs}$. Thin sections were mounted on grids and examined under the electron microscope (Philips EM410).

\section{Autophagy flux assays mCherry-GFP-LC3 transfection}

Cat $\mathrm{S}^{-/-}$or WT BMDMs were transfected with mCherryGFP-LC3 reporter construct by using Lipofectamine 2000
(Invitrogen, Carlsbad, CA) as previously described in our lab [31]. After transfection, Cat $\mathrm{S}^{-/-}$or WT BMDMs cocultured with SL4 cells without serum for $48 \mathrm{hrs}$. Cells were fixed with $4 \%$ paraformaldehyde and microphotographs of mCherry-GFP-LC3 fluorescence were obtained with the confocal laser-scanning microscope (TCS 4D; Leica, Heidelberg, Germany). Treatment-induced changes in GFP intensity in mCherry-positive puncta were calculated to depict autophagic flux.

\section{High-throughput image analysis}

Cat $\mathrm{S}^{-/-}$or WT BMDMs expressing GFP-LC3 were seeded in 96-well plates, then cocultured with SL4 cells in $100 \mu \mathrm{l}$ of medium/well without serum for $48 \mathrm{hrs}$. Images data were collected with an ArrayScan HCS 4.0 Reader with a $20 \times$ objective (Cellomics) for hoechst-labeled nuclei and GFP-tagged LC3. The detection of punctated staining of GFP-LC3 from the diffuse staining indicated the formation of autophagosomes. Images of 1,000 cells for each well were analyzed to obtain the average cell number per field, fluorescence spot number, area, and intensity per cell.

\section{DQ-BSA degradation assays}

To quantify protein degradation induced by stimuli, autophagy flux was analyzed by flow cytometry and confocal microscopy using the self-quenched substrate DQ-Green BSA (Molecular Probes, Eugene, OR) as described in previous studies [59]. Briefly, WT or Cat $\mathrm{S}^{-/-}$BMDMs were cocultured with SL4 cells for $48 \mathrm{hrs}$ and serum-starved for at least $12 \mathrm{hrs}$ before co-culture. WT BMDMs were in the 
presence or absence of Cat $\mathrm{S}$ inhibitor Z-FL-COCHO $(10 \mu \mathrm{mol} / \mathrm{L})$ cocultured with SL4 cells. WT or Cat $\mathrm{S}^{-/-}$ BMDMs into the lower compartment were loaded with $10 \mu \mathrm{g} / \mathrm{ml} \mathrm{DQ-Green} \mathrm{BSA} \mathrm{for} 15 \mathrm{~min}$ at $37^{\circ} \mathrm{C}$. The cells were washed twice with PBS to remove excess label, then harvested at indicated time points. Cells were harvested, and Green-fluorescent of DQ-BSA was analyzed by flow cytometry using a FACSCalibur flow cytometer (BD Biosciences) and CellQuest (BD Biosciences) and FlowJo (Treestar) software. For confocal images analysis, cells were placed on coverslips after treatment as above and fixed with $4 \%$ formaldehyde. The fluorescent degradation products of DQ-BSA in lysosomes were imaged using a confocal laser-scanning microscope (TCS 4D; Leica, Heidelberg, Germany).

\section{Statistics}

Data were analyzed with GraphPad Prism v5.00 for Windows. Results are expressed as mean \pm SEM. Differences were analyzed by $t$ test or ANOVA, and results were considered significant at a $P<0.05$.

\section{Additional files}

Additional file 1: Figure S1. Cat S deficiency inhibits angiogenesis in tumor development. The area of vessels was evaluated by immunohistochemical analysis with anti-CD31 antibody in $\mathrm{PancO} 2$ subcutaneous tumors (A) and in metastasized foci after intrasplenic injection of SL4 cells (B). ( $\times 200$ magnification and Scale bars $=100 \mu \mathrm{m})$. Quantitative analysis of CD31 expression in subcutaneous tumors and metastasized foci sections. Data are mean \pm SEM for $n=8$ mice with 10 fields per animal. ${ }^{*}, P<0.01$.

Additional file 2: Figure S2. SL4 cells induce Cat S expression in macrophages. (A) BMDMs were cocultured with SL4 cells for 48 hrs and serum-starved for at least 12 hrs before co-culture. Double immunofluorescence analyses of Cat $\mathrm{S}$ expression in BMDMs with or without SL4 cells treatment. BMDMs were stained with anti-F4/80 (red) or anti-Cat S (green) antibody and DAPI (blue; to stain the nuclei). ( $\times 400$ magnification and Scale bars $=50 \mu \mathrm{m}$ ). Three independent experiments were performed. (B) Western blot analysis of the protein levels of Cat S in BMDMs with or without SL4 cells treatment. GAPDH was used as a loading control. Quantitative analysis of Cat S/GAPDH ratio in BMDMs with or without SL4 cells treatment. Data are mean \pm SEM of 3 independent experiments. ${ }^{*}, P<0.01$.

\footnotetext{
Abbreviations

Cat S: Cathepsin S; Cat B: Cathepsin B; Cat D: Cathepsin D; Cat F: Cathepsin F; Cat H: Cathepsin H; Cat K: Cathepsin K; Cat L: Cathepsin L; Cat Z: Cathepsin Z; TAMs: Tumor-associated macrophages; Arg-1: Arginase-1; FIZZ1: Found in inflammatory zone protein; IL-10: Interleukin-10; iNOS: Inducible nitric oxide synthase; IL-1 $\beta$ : Interleukin-1 $\beta$; TNF-a: Tumor necrosis factor $a$; CQ: Chloroquine.
}

\section{Competing interests}

The authors declare that there are no conflicts of interest.

\section{Authors' contributions}

MY and $J$ equally participated in the design and execution of the overall study. JS carried out the pathologic analysis. YQ provided Cat S knockout mice and performed mice genotyping. QJ, XZ participated in its coordination and provided technical support. JD was involved in the conception and design of the study as well as in revising the manuscript. All authors read and approved the final manuscript.

\section{Acknowledgments}

This study was supported by grants from Chinese Ministry of Science and Technology (2009CB522205), Chinese High Technology Research and Development Program (2012AA02A201), National Science Foundation of China (81170120) and Beijing Nova Program (Z121107002512041).

\section{Author details}

${ }^{1}$ Beijing Anzhen Hospital, Capital Medical University, Beijing, China. ${ }^{2} T$ he Key Laboratory of Remodeling-Related Cardiovascular Diseases, Capital Medical University, Ministry of Education, Beijing Institute of Heart Lung and Blood Vessel Diseases, Beijing 100029, China. ${ }^{3}$ The Second Affiliated Hospital to Nanchang University, Jiangxi 330006, China. ${ }^{4}$ nnovation Center China, AstraZeneca, Shanghai 201203, China.

Received: 30 October 2013 Accepted: 19 February 2014

Published: 2 March 2014

\section{References}

1. Albini A, Sporn MB: The tumour microenvironment as a target for chemoprevention. Nat Rev Cancer 2007, 7:139-147.

2. de Visser KE, Coussens LM: The inflammatory tumor microenvironment and its impact on cancer development. Contrib Microbiol 2006, 13:118-137.

3. Mantovani A, Allavena P, Sica A, Balkwill F: Cancer-related inflammation. Nature 2008, 454:436-444.

4. Mantovani A, Sozzani S, Locati M, Allavena P, Sica A: Macrophage polarization: tumor-associated macrophages as a paradigm for polarized M2 mononuclear phagocytes. Trends Immunol 2002, 23:549-555.

5. Mantovani A, Sica A: Macrophages, innate immunity and cancer: balance, tolerance, and diversity. Curr Opin Immunol 2010, 22:231-237.

6. Condeelis J, Pollard JW: Macrophages: obligate partners for tumor cell migration, invasion, and metastasis. Cell 2006, 124:263-266.

7. Qian BZ, Pollard JW: Macrophage diversity enhances tumor progression and metastasis. Cell 2010, 141:39-51.

8. Shabo I, Stal O, Olsson H, Dore S, Svanvik J: Breast cancer expression of CD163, a macrophage scavenger receptor, is related to early distant recurrence and reduced patient survival. Int I Cancer 2008, 123:780-786.

9. Kurahara H, Shinchi H, Mataki Y, Maemura K, Noma H, Kubo F, Sakoda M, Ueno S, Natsugoe S, Takao S: Significance of M2-polarized tumor-associated macrophage in pancreatic cancer. J Surg Res 2011, 167:e211-219.

10. Komohara Y, Ohnishi K, Kuratsu J, Takeya M: Possible involvement of the M2 anti-inflammatory macrophage phenotype in growth of human gliomas. J Pathol 2008, 216:15-24.

11. Mizushima N, Levine B, Cuervo AM, Klionsky DJ: Autophagy fights disease through cellular self-digestion. Nature 2008, 451:1069-1075.

12. Kroemer $G$, Marino G, Levine B: Autophagy and the integrated stress response. Mol Cell 2010, 40:280-293.

13. Yang Z, Klionsky DJ: Mammalian autophagy: core molecular machinery and signaling regulation. Curr Opin Cell Biol 2010, 22:124-131.

14. Levine B, Kroemer G: Autophagy in the pathogenesis of disease. Cell 2008, $132: 27-42$.

15. Levine B, Yuan J: Autophagy in cell death: an innocent convict? J Clin Invest 2005, 115:2679-2688

16. Maiuri MC, Zalckvar E, Kimchi A, Kroemer G: Self-eating and self-killing: crosstalk between autophagy and apoptosis. Nat Rev Mol Cell Biol 2007, 8:741-752.

17. Kondo $Y$, Kanzawa $T$, Sawaya R, Kondo $\mathrm{S}$ : The role of autophagy in cancer development and response to therapy. Nat Rev Cancer 2005, 5:726-734.

18. Kenific CM, Thorburn A, Debnath J: Autophagy and metastasis: another double-edged sword. Curr Opin Cell Biol 2010, 22:241-245.

19. Mathew R, Karantza-Wadsworth V, White E: Role of autophagy in cancer. Nat Rev Cancer 2007, 7:961-967.

20. Mohamed MM, Sloane BF: Cysteine cathepsins: multifunctional enzymes in cancer. Nat Rev Cancer 2006, 6:764-775.

21. Gong F, Peng X, Luo C, Shen G, Zhao C, Zou L, Li L, Sang Y, Zhao Y, Zhao X: Cathepsin $B$ as a potential prognostic and therapeutic marker for human lung squamous cell carcinoma. Mol Cancer 2013, 12:125.

22. Withana NP, Blum G, Sameni M, Slaney C, Anbalagan A, Olive MB, Bidwell BN, Edgington L, Wang L, Moin K, Sloane BF, Anderson RL, Bogyo MS, 
Parker BS: Cathepsin B inhibition limits bone metastasis in breast cancer. Cancer Res 2012, 72:1199-1209.

23. Gocheva V, Zeng W, Ke D, Klimstra D, Reinheckel T, Peters C, Hanahan D, Joyce JA: Distinct roles for cysteine cathepsin genes in multistage tumorigenesis. Genes Dev 2006, 20:543-556.

24. Flannery T, Gibson D, Mirakhur M, McQuaid S, Greenan C, Trimble A, Walker B, McCormick D, Johnston PG: The clinical significance of cathepsin S expression in human astrocytomas. Am J Pathol 2003, 163:175-182.

25. Burden RE, Gormley JA, Jaquin TJ, Small DM, Quinn DJ, Hegarty SM, Ward C, Walker B, Johnston JA, Olwill SA, Scott CJ: Antibody-mediated inhibition of cathepsin $\mathrm{S}$ blocks colorectal tumor invasion and angiogenesis. Clin Cancer Res 2009, 15:6042-6051.

26. Shi GP, Webb AC, Foster KE, Knoll JH, Lemere CA, Munger JS, Chapman HA: Human cathepsin S: chromosomal localization, gene structure, and tissue distribution. J Biol Chem 1994, 269:11530-11536.

27. Ryschich E, Lizdenis $P$, Ittrich C, Benner A, Stahl S, Hamann A, Schmidt J, Knolle P, Arnold B, Hammerling GJ, Ganss R: Molecular fingerprinting and autocrine growth regulation of endothelial cells in a murine model of hepatocellular carcinoma. Cancer Res 2006, 66:198-211.

28. Flannery T, McQuaid S, McGoohan C, McConnell RS, McGregor G, Mirakhur M, Hamilton P, Diamond J, Cran G, Walker B, Scott C, Martin L, Ellison D, Patel C, Nicholson C, Mendelow D, McCormick D, Johnston PG: Cathepsin S expression: an independent prognostic factor in glioblastoma tumours-A pilot study. Int J Cancer 2006, 119:854-860.

29. Flannery T, McConnell RS, McQuaid S, McGregor G, Mirakhur M, Martin L, Scott C, Burden R, Walker B, McGoohan C, Johnston PG: Detection of cathepsin $\mathrm{S}$ cysteine protease in human brain tumour microdialysates in vivo. Br J Neurosurg 2007, 21:204-209.

30. Gocheva V, Wang HW, Gadea BB, Shree T, Hunter KE, Garfall AL, Berman T, Joyce JA: IL-4 induces cathepsin protease activity in tumor-associated macrophages to promote cancer growth and invasion. Genes Dev 2010, 24:241-255.

31. Pan L, Li Y, Jia L, Qin Y, Qi G, Cheng J, Qi Y, Li H, Du J: Cathepsin S deficiency results in abnormal accumulation of autophagosomes in macrophages and enhances Ang II-induced cardiac inflammation. PLoS One 2012, 7:e35315.

32. Morimoto-Tomita M, Ohashi Y, Matsubara A, Tsuiji M, Irimura T: Mouse colon carcinoma cells established for high incidence of experimental hepatic metastasis exhibit accelerated and anchorage-independent growth. Clin Exp Metastasis 2005, 22:513-521.

33. Solinas G, Germano G, Mantovani A, Allavena P: Tumor-associated macrophages (TAM) as major players of the cancer-related inflammation. J Leukoc Biol 2009, 86:1065-1073.

34. Kimmelman AC: The dynamic nature of autophagy in cancer. Genes Dev 1999-2010, 2011:25.

35. Li L, Ishdorj G, Gibson SB: Reactive oxygen species regulation of autophagy in cancer: implications for cancer treatment. Free Radic Biol Med 2012, 53:1399-1410.

36. Sakaki K, Wu J, Kaufman RJ: Protein kinase Ctheta is required for autophagy in response to stress in the endoplasmic reticulum. J Biol Chem 2008, 283:15370-15380.

37. Iwai-Kanai E, Yuan H, Huang C, Sayen MR, Perry-Garza CN, Kim L, Gottlieb RA: A method to measure cardiac autophagic flux in vivo. Autophagy 2008, 4:322-329

38. Schonteich E, Wilson GM, Burden J, Hopkins CR, Anderson $\mathrm{K}$, Goldenring JR, Prekeris R: The Rip11/Rab11-FIP5 and kinesin II complex regulates endocytic protein recycling. J Cell Sci 2008, 121:3824-3833.

39. Gormley JA, Hegarty SM, O'Grady A, Stevenson MR, Burden RE, Barrett HL, Scott CJ, Johnston JA, Wilson RH, Kay EW, Johnston PG, Olwill SA: The role of Cathepsin $\mathrm{S}$ as a marker of prognosis and predictor of chemotherapy benefit in adjuvant CRC: a pilot study. Br J Cancer 2011, 105:1487-1494.

40. Kos J, Sekirnik A, Kopitar G, Cimerman N, Kayser K, Stremmer A, Fiehn W, Werle B: Cathepsin $\mathrm{S}$ in tumours, regional lymph nodes and sera of patients with lung cancer: relation to prognosis. Br J Cancer 2001, 85:1193-1200.

41. Lindahl C, Simonsson M, Bergh A, Thysell E, Antti H, Sund M, Wikstrom P: Increased levels of macrophage-secreted cathepsin $\mathrm{S}$ during prostate cancer progression in TRAMP mice and patients. Cancer Genomics Proteomics 2009, 6:149-159.

42. Wang B, Sun J, Kitamoto S, Yang M, Grubb A, Chapman HA, Kalluri R, Shi GP: Cathepsin $\mathrm{S}$ controls angiogenesis and tumor growth via matrix-derived angiogenic factors. J Biol Chem 2006, 281:6020-6029.
43. Gillet L, Roger S, Besson P, Lecaille F, Gore J, Bougnoux P, Lalmanach G, Le Guennec JY: Voltage-gated Sodium Channel Activity Promotes Cysteine Cathepsin-dependent Invasiveness and Colony Growth of Human Cancer Cells. J Biol Chem 2009, 284:8680-8691.

44. Kwok HF, Buick RJ, Kuehn D, Gormley JA, Doherty D, Jaquin TJ, McClurg A, Ward C, Byrne T, Jaworski J, Leung KL, Snoddy P, McAnally C, Burden RE, Gray B, Lowry J, Sermadiras I, Gruszka N, Courtenay-Luck N, Kissenpfennig A, Scott CJ, Johnston JA, Olwill SA: Antibody targeting of Cathepsin S induces antibody-dependent cellular cytotoxicity. Mol Cancer 2011, 10:147. doi: 10.1186/1476-4598-10-147.

45. Verdoes M, Edgington LE, Scheeren FA, Leyva M, Blum G, Weiskopf K, Bachmann MH, Ellman JA, Bogyo M: A nonpeptidic cathepsin S activity-based probe for noninvasive optical imaging of tumor-associated macrophages. Chem Biol 2012, 19:619-628.

46. Shree T, Olson OC, Elie BT, Kester JC, Garfall AL, Simpson K, Bell-McGuinn KM, Zabor EC, Brogi E, Joyce JA: Macrophages and cathepsin proteases blunt chemotherapeutic response in breast cancer. Genes Dev 2011, 25:2465-2479.

47. Small DM, Burden RE, Jaworski J, Hegarty SM, Spence S, Burrows JF, McFarlane C, Kissenpfennig A, McCarthy HO, Johnston JA, Walker B, Scott $\mathrm{CJ}$ : Cathepsin S from both tumor and tumor-associated cells promote cancer growth and neovascularization. Int J Cancer 2013, 133:2102-2112.

48. Ward C, Kuehn D, Burden RE, Gormley JA, Jaquin TJ, Gazdoiu M, Small D, Bicknell R, Johnston JA, Scott CJ, Olwill SA: Antibody targeting of cathepsin $S$ inhibits angiogenesis and synergistically enhances anti-VEGF. PLOS One 2010, 5:e12543.

49. Liu J, Sukhova GK, Sun JS, Xu WH, Libby P, Shi GP: Lysosomal cysteine proteases in atherosclerosis. Arterioscler Thromb Vasc Biol 2004, 24:1359-1366.

50. Turk V, Stoka V, Vasiljeva O, Renko M, Sun T, Turk B, Turk D: Cysteine cathepsins: from structure, function and regulation to new frontiers. Biochim Biophys Acta 1824, 2012:68-88.

51. Schonthal AH: Endoplasmic reticulum stress and autophagy as targets for cancer therapy. Cancer Lett 2009, 275:163-169.

52. Bialik S, Kimchi A: Autophagy and tumor suppression: recent advances in understanding the link between autophagic cell death pathways and tumor development. Adv Exp Med Biol 2008, 615:177-200.

53. Kaminskyy V, Zhivotovsky B: Proteases in autophagy. Biochim Biophys Acto 1824, 2011:44-50

54. Gupta S, Singh RK, Dastidar S, Ray A: Cysteine cathepsin S as an immunomodulatory target: present and future trends. Expert Opin Ther Targets 2008, 12:291-299.

55. Driessen C, Bryant RA, Lennon-Dumenil AM, Villadangos JA, Bryant PW, Shi GP, Chapman HA, Ploegh HL: Cathepsin S controls the trafficking and maturation of MHC class II molecules in dendritic cells. J Cell Biol 1999, 147:775-790.

56. Stoeckle C, Quecke P, Ruckrich T, Burster T, Reich M, Weber E, Kalbacher $H$, Driessen C, Melms A, Tolosa E: Cathepsin S dominates autoantigen processing in human thymic dendritic cells. J Autoimmun 2012, 38:332-343.

57. Morikane K, Tempero RM, Sivinski CL, Nomoto M, Van Lith ML, Muto T, Hollingsworth MA: Organ-specific pancreatic tumor growth properties and tumor immunity. Cancer Immunol Immunother 1999, 47:287-296.

58. Yang M, Zheng J, Miao Y, Wang Y, Cui W, Guo J, Qiu S, Han Y, Jia L, Li H, Cheng J, Du J: Serum-glucocorticoid regulated kinase 1 regulates alternatively activated macrophage polarization contributing to angiotensin II-induced inflammation and cardiac fibrosis. Arterioscler Thromb Vasc Biol 2012, 32:1675-1686.

59. Hetz C, Thielen P, Matus S, Nassif M, Court F, Kiffin R, Martinez G, Cuervo AM, Brown RH, Glimcher LH: XBP-1 deficiency in the nervous system protects against amyotrophic lateral sclerosis by increasing autophagy. Genes Dev 2009, 23:2294-2306.

\section{doi:10.1186/1476-4598-13-43}

Cite this article as: Yang et al:: Cathepsin S-mediated autophagic flux in tumor-associated macrophages accelerate tumor development by promoting M2 polarization. Molecular Cancer 2014 13:43. 